\title{
Efecto del consumo de hidratos de carbono en los deportes de resistencia
}

\section{Effect of carbohydrate consumption on endurance sports}

\author{
LÓPEZ-SOLÍS, Ricardo†', CAMACHO-DÍAZ, Brenda Hildeliza”, CAMPOS-MENDIOLA, Roberto” \\ y ORTÍZ-RODRÍGUEZ, María Araceli*'
}

'Facultad de Nutrición, Universidad Autónoma del Estado de Morelos (UAEM). Avenida Universidad 1001, Chamilpa, 62209. Cuernavaca, Mor.

"Instituto Politécnico Nacional-Centro de Desarrollo de Productos Bióticos (IPN-CEPROBI). Carr Yautepec - Jojutla s/n, San Isidro, Mor.

ID $1^{\text {er }}$ Autor: Ricardo López-Solís / ORC ID: 0000-0002-6619-8772, CVU CONACYT ID: 922259

ID $1^{\text {er }}$ Coautor: Brenda Hildeliza, Camacho-Díaz / ORC ID: 0000-0001-5562-0782, Researcher ID Thomson: C-10342018, CVU CONACYT ID: 205272

ID 2 ${ }^{\text {do }}$ Coautor: Roberto, Campos-Mendiola / ORC ID: 0000-0002-7810-2459, CVU CONACYT ID: 210184

ID $3^{\text {er }}$ Coautor: María Araceli, Ortiz-Rodríguez / ORC ID: 0000-0003-0847-0261, Researcher ID Thomson: T-37072018, CVU CONACYT ID: 449164

DOI: $10.35429 / J P D .2019 .9 .3 .1 .7$

Recibido 12 de Julio, 2019; Aceptado 28 de Septiembre, 2019

\begin{abstract}
Resumen
El objetivo de esta revisión es resumir los efectos del consumo de hidratos de carbono (HCO) en los deportes de larga duración. Debido a la promoción de la actividad física por parte de las autoridades en salud, la participación en los deportes de resistencia va en aumento. Independientemente del nivel, los atletas buscan formas de optimizar su rendimiento mediante el entrenamiento y la nutrición. En consecuencia, la investigación en nutrición deportiva se ha centrado en aquellas estrategias que pueden mejorar el rendimiento. La fatiga durante el ejercicio prolongado se asocia con depleción de glucógeno muscular y reducción de las concentraciones de glucosa en sangre. Por lo tanto, la alimentación con $\mathrm{HCO}$ durante el ejercicio ha demostrado que puede mejorar la capacidad y el rendimiento, debido a que ayuda a mantener los niveles de glucosa en sangre, así como, a aumentar las tasas de oxidación de hidratos de carbono y preservar el glucógeno, encontrándose mejores resultados cuando se consumen HCO dependientes de distintos transportadores intestinales para su absorción (p.ej. fructosa y glucosa), ya que de esta forma es posible aumentar las tasas de absorción, el vaciado gástrico y la absorción de líquidos, además de disminuir las molestias gastrointestinales.
\end{abstract}

Deportes de resistencia, hidratos de carbono, nutrición deportiva

Citación: LÓPEZ-SOLÍS, Ricardo, CAMACHO-DÍAZ, Brenda Hildeliza, CAMPOS-MENDIOLA, Roberto y ORTÍZRODRÍGUEZ, María Araceli. Efecto del consumo de hidratos de carbono en los deportes de resistencia. Revista de Didáctica Práctica. 2019. 3-9: 1-7.

\begin{abstract}
The aim of this review is to summarize the effects of carbohydrate consumption on endurance sports. Due to the promotion of physical activity by health authorities, participation in endurance sports is increasing. Regardless of the level, athletes look for ways to optimize their performance through training and nutrition. In consequence, research in the field of sports nutrition has focused on those strategies that can improve performance. Fatigue during prolonged exercise is associated with depletion of muscle glycogen and reduced blood glucose concentrations. Therefore, carbohydrate feeding during exercise has shown that it can improve capacity and performance, because it helps maintain blood glucose levels, as well as, increase carbohydrate oxidation rates and preserve glycogen, finding better results when are consumed multipletranportable carbohydrates for absortion (e.g., fructose and glucose), since in this way is possible to increase absorption rates, gastric emptying and fluid absorption, in addition to decrease gastrointestinal distress.
\end{abstract}

Endurance sports, carbohydrates, sport nutrition

\footnotetext{
*Correspondencia al Autor (Correo Electrónico: araceli.ortiz@gmail.com)

$\uparrow$ Investigador contribuyendo como primer autor.
} 


\section{Introducción}

Actualmente la promoción de la actividad físico-deportiva es considerada como uno de los objetivos prioritarios para las autoridades en salud pública, como una herramienta para combatir la pandemia de enfermedades asociadas al síndrome metabólico y al sedentarismo (Instituto Nacional de Estadística y Geografía, 2013; Díaz, Rodríguez \& Rodríguez, 2017). Por lo que muchos eventos son organizados para alentar a las personas a practicar deportes de resistencia (Jeukendrup, 2011), lo que se ve reflejado en el número creciente de competencias. Como ejemplo, en México el número de carreras atléticas ha aumentado un $34 \%$ desde el 2015, pasando de 1,552 a 2,078 en el 2018 (Run México, 2019). Para el propósito de esta revisión, nos referiremos como deporte de resistencia a aquellos en los que la causa de la fatiga sea el agotamiento del sustrato, por lo general deportes con una duración de 30 min o más (Saris et al., 2003).

Independientemente de su nivel, los atletas buscan formas de mejorar su rendimiento mediante el entrenamiento y la nutrición (Jeukendrup, 2011). En consecuencia, la investigación en nutrición deportiva se ha centrado en aquellas estrategias que pueden mejorar el rendimiento (Close, Hamilton, Philp, Burke \& Morton, 2016).

En los deportes de resistencia una estrategia para mejorar el rendimiento cuyos beneficios están bien establecidos es el consumo de hidratos de carbono (HCO) (Campbell et al., 2013; Close et al., 2016; Jeukendrup, 2011; Ormsbee, Bach \& Baur, 2014; Wilson, 2015; Zhang, O’Kennedy \& Morton, 2015), por lo que muchos suplementos deportivos con $\mathrm{HCO}$ se comercializan y se venden en todo el mundo, incluidas bebidas, geles, semisólidos, polvos y otros productos(Wilson, 2015) y son ampliamente utilizados por los atletas de cualquier nivel como una ayuda ergogénica antes y durante los eventos deportivos (Pöchmüller, Schwingshackl, Colombani \& Hoffmann, 2016; Wilson, 2015). Por lo que el objetivo de esta revisión será describir y explicar los efectos del consumo de $\mathrm{HCO}$, específicamente durante el ejercicio de resistencia.

\section{Importancia y función de los hidratos de carbono en el ejercicio}

Los $\mathrm{HCO}$ son considerados la principal fuente de energía del organismo y la más rápida, aportando del 40 al $80 \%$ de los requerimientos totales de energía. Junto con los lípidos y las proteínas se encargan de mantener al organismo, estructural y funcionalmente, tanto en reposo como durante la actividad física, cumpliendo ciertas funciones relacionadas al metabolismo energético y al desempeño físico (Peniche, 2011).

La función más importante de los $\mathrm{HCO}$ en los deportes de resistencia es el suministro de energía para las células musculares. La alimentación con HCO durante el ejercicio ha demostrado que puede mejorar la capacidad y el rendimiento, ya que permite retrasar la fatiga asociada con la reducción de la glucosa en sangre y la depleción de glucógeno muscular, principal almacén de glucosa del organismo (Jeukendrup, 2011; Peinado, Rojo-Tirado \& Benito, 2013; Peniche, 2011; Fuchs, Gonzalez \& van Loon, 2019).

\section{Fuentes de energía en el deporte de resistencia}

Para poder ser utilizada por las fibras musculares, la energía contenida en los alimentos necesita ser transformada de energía química a energía mecánica mediante la oxidación de los macronutrimentos (Peniche, 2011).

La energía requerida para la contracción muscular durante el ejercicio se obtiene a partir del adenosín trifosfato (ATP) resultante del metabolismo de los HCO, lípidos y proteínas. Cuando las fibras musculares se contraen y activan la enzima trifosfatasa de adenosina (ATP-asa), uno de los fosfatos de la molécula de ATP se desprende y libera energía de su ligadura, dando como resultado un difosfato de adenosina (ADP) y $7.3 \mathrm{kcal}$ que se liberan de la rotura de la ligadura (Bompa \& Buzzichelli, 2015; Peniche, 2011).

La cantidad de ATP almacenada en el músculo es limitada y por tanto se debe resintetizar continuamente para permitir el proceso del trabajo muscular. El cuerpo humano puede producir y reciclar ATP a través de tres procesos diferentes pero que actúan de manera conjunta según la demanda del ejercicio: 
1) Sistema de los fosfágenos (ATP-PC) o anaeróbico aláctico; 2) Sistema del ácido láctico o glucolisis anaerobia; y 3) Sistema aeróbico o sistema oxidativo (Bompa \& Buzzichelli, 2015; Peniche, 2011).

En los deportes de resistencia más del $85 \%$ de la energía proviene del sistema aeróbico, siendo mayor el porcentaje de contribución de este sistema conforme aumenta la duración del ejercicio. Las reservas de glucógeno muscular y la glucosa sanguínea son los sustratos más importantes como fuente de energía rápida para el organismo, sin embargo, después de aproximadamente $90 \mathrm{~min}$ de ejercicio moderado a intenso esta reservas pueden disminuirse hasta en un 40-60\%, por lo que el ejercicio prolongado ( $\geq 2$ horas) puede dar lugar a la degradación de las grasas y las proteínas para la producción de ATP (Fuchs et al., 2019; Bompa \& Buzzichelli, 2015; Peinado, 2013).

Los depósitos bajos de glucógeno pueden contribuir a la fatiga, reduciendo así la capacidad de ejercicio de resistencia, ya que el rendimiento de energía por volumen de oxígeno es menor con la utilización de las grasas respecto a los HCO como combustible energético (Fuchs et al., 2019). Por lo que la intervención nutricional más beneficiosa será aquella que pueda aumentar o conservar por más tiempo las reservas de glucógeno muscular y hepático. Las estrategias que permiten lograr esto son: 1) proporcionar $\mathrm{HCO}$ exógenos para servir como fuente de combustible; 2) alterar la utilización del sustrato de una manera que preserve los HCO endógenos; o 3) una combinación de las dos (Close et al., 2016; Ormsbee et al., 2014). Siendo de interés para esta revisión la primera de estas estrategias.

\section{Consumo de hidratos de carbono durante el ejercicio de resistencia}

Como se mencionó anteriormente, la alimentación con $\mathrm{HCO}$ durante el ejercicio permite retrasar el agotamiento del glucógeno muscular, mantener los niveles de glucosa en sangre y por lo tanto altos niveles de oxidación de HCO, postergando así la fatiga y mejorando el rendimiento (Jeukendrup, 2011; Peinado et al., 2013; Peniche, 2011; Campbell et al., 2013).
La cantidad y el tipo de $\mathrm{HCO}$ recomendada es en función de la duración del ejercicio. En eventos con una duración menor a una hora el efecto del consumo de $\mathrm{HCO}$ es similar a solo enjuagarse la boca con una bebida de HCO, lo que significa que los $\mathrm{HCO}$ actúan estimulando el sistema nervioso central y no necesariamente como fuente energética. Por otro lado, cuando el ejercicio es más prolongado ( $\geq 2$ horas), la ingesta de $\mathrm{HCO}$ exógenos como fuente de energía es esencial (Jeukendrup, 2011). Investigaciones recientes indican que la concentración, el tipo y / o la composición de $\mathrm{HCO}$ consumidos pueden influir en el vaciamiento gástrico, la administración de líquidos, la absorción y el malestar gastrointestinal (Ormsbee et al., 2014). En la tabla 1 se muestran las cantidades de HCO recomendadas según la duración del ejercicio, más adelante en el texto se explica con mayor detalle el tipo de HCO.

\section{Absorción intestinal y tasas de oxidación de distintos hidratos de carbono}

La oxidación de HCO provenientes de la dieta durante el ejercicio está condicionada por la absorción intestinal de los mismos, que a su vez responde al vaciado gástrico y al número y actividad de transportadores intestinales (Ormsbee et al., 2014; Jeukendrup, 2011). Los HCO exógenos se absorben principalmente en el intestino delgado, mayoritariamente en el duodeno y el yeyuno, y se transportan a través del epitelio intestinal por medio de transporte activo y facilitado (Kellett, Brot-Laroche, Mace \& Leturque, 2008). A continuación se describe la absorción intestinal y las tasas de oxidación de distintos HCO:

Glucosa: se absorbe por transporte activo en el enterocito a través de una proteína transportadora dependiente de sodio llamada SGLT1, esta proteína transportadora se satura cuando se ingiere glucosa a una velocidad de 1 $\mathrm{g} / \mathrm{min}$. Una vez en el enterocito, la glucosa atraviesa la membrana basolateral hacia la circulación a través del transportador facilitador GLUT2 (Ver figura 1) (Jeukendrup, 2013; Jeukendrup, 2011; Kellett et al., 2008). 
Galactosa: utiliza los mismos transportadores que la glucosa para su absorción (SGLT1 y GLUT2), sin embargo, sus tasas de oxidación son casi $50 \%$ más bajas, ya que debe convertirse en glucosa en el hígado antes de que pueda oxidarse en el músculo (Ver figura 1) (Jeukendrup, 2013; Peniche, 2011).

Fructosa: es absorbida por un transportador independiente de sodio llamado GLUT5, las tasas máximas de absorción de este monosacárido oscilan alrededor de $0.6 \mathrm{~g} / \mathrm{min}$, al igual que con los dos monosacáridos mencionados anteriormente, el GLUT2 le proporciona una vía de salida del enterocito a la circulación sanguínea, y como en el caso de la galactosa debe ser convertida en glucosa en el hígado, o en menor proporción en los riñones, para ser utilizada por el músculo como fuente de energía (Ver figura 1) (Jeukendrup, 2013; Kellett et al., 2008; Tappy \& Rosset, 2017).

\begin{tabular}{|l|l|l|}
\hline \multicolumn{1}{|c|}{$\begin{array}{c}\text { Duración } \\
\text { del ejercicio }\end{array}$} & \multicolumn{1}{|c|}{$\begin{array}{c}\text { Ingesta } \\
\text { recomendada }\end{array}$} & \multicolumn{1}{|c|}{$\begin{array}{c}\text { Tipo de HCO } \\
\text { recomendados } \\
\text { (STC o MTC) }\end{array}$} \\
\hline$<30 \mathrm{~min}$ & No se requiere & - \\
\hline $30-75 \mathrm{~min}$ & Enjuague bucal & STC o MTC \\
\hline $1-2$ horas & Hasta $30 \mathrm{~g} /$ hora & STC o MTC \\
\hline $2-3$ horas & Hasta $60 \mathrm{~g} /$ hora & $\begin{array}{l}\text { Preferentemente } \\
\text { MTC }\end{array}$ \\
\hline >2.5 horas & Hasta $90 \mathrm{~g} /$ hora & MTC \\
\hline $\begin{array}{l}\text { Nota: Estas pautas están destinadas a ejercicio de } \\
\text { intensidad razonable ( }>4 \mathrm{kcal} / \text { min). Si la intensidad } \\
\text { es menor, las cantidades de ingesta deben ajustarse } \\
\text { hacia abajo. HCO= Hidratos de carbono; MTC= } \\
\text { Hidratos de carbono de múltiples transportadores; } \\
\text { STC= Hidratos de carbono de transportador único }\end{array}$ \\
\hline
\end{tabular}

Tabla 1 Recomendaciones de consumo de hidratos de carbono según la duración del ejercicio

Fuente: Jeukendrup, 2011

Maltosa y polímeros de glucosa: la maltosa (2 moléculas de glucosa) y los polímeros de glucosa (maltodextrina y amilopectina), se comportan de manera idéntica a la glucosa, lo que indica que la hidrólisis, que tiene lugar en la cavidad oral y los intestinos, no es un factor limitante para la absorción y oxidación (Jeukendrup, 2013).

Sacarosa: este HCO parece dar altas tasas de oxidación, similares a la glucosa, a pesar de que su hidrolisis da como resultado una molécula de glucosa y una de fructosa, siendo que esta última tiene tasas más bajas de oxidación (Jeukendrup, 2013).
Otros HCO: la amilosa (polímero de glucosa), así como otros HCO menos comunes, como la isomaltulosa (glucosa + fructosa) y la trehalosa (2 moléculas de glucosa) tienen tasas de oxidación bajas (Jeukendrup, 2013).

En resumen, los $\mathrm{HCO}$ se pueden dividir en dos grupos: 1) HCO que se oxidan rápidamente (hasta $\sim 60 \mathrm{~g} /$ hora $=1 \mathrm{~g} / \mathrm{min}$ ) como la glucosa, maltosa, sacarosa, maltodextrina y amilopectina; 2) HCO que se oxidan relativamente lento (hasta $\sim 30 \mathrm{~g} /$ hora $=0.5 \mathrm{~g} / \mathrm{min}$ ) como la fructosa, galactosa, isomaltulosa, trehalosa y amilosa (Jeukendrup, 2013).

\section{Diferencias individuales en la oxidación de hidratos de carbono}

Las diferencias individuales en la oxidación de $\mathrm{HCO}$ exógenos son relativamente pequeñas. La masa corporal no ha mostrado correlación con la oxidación exógena de HCO, lo cual posiblemente se debe a que el factor limitante de la oxidación es la absorción y esta es independiente de la masa corporal. Por lo que probablemente las pequeñas variaciones encontradas en la oxidación de HCO se deban a la capacidad del intestino para la absorción de los mismos. Se ha demostrado que la capacidad de absorción esta relacionada con la cantidad de HCO consumidos habitualmente en la dieta, en otras palabras, una dieta alta en $\mathrm{HCO}$ aumentará la cantidad y la actividad de los transportadores SGLT1 en el intestino, permitiendo una mayor absorción y oxidación de HCO durante el ejercicio (Jeukendrup, 2013; Stellingwerff \& Cox, 2014; Jeukendrup, 2017).

\section{Efectos del consumo combinado de hidratos de carbono dependientes de distintos transportadores intestinales}

Mientras que generalmente se aceptaba que las tasas de oxidación de HCO exógenos se consideraban limitadas a aproximadamente $1 \mathrm{~g}$ / min debido a la saturación de los transportadores de glucosa intestinal (SGLT1), ahora se sabe que las tasas de oxidación pueden alcanzar hasta 1,8 g / min con la mezcla de $\mathrm{HCO}$ dependientes de distintos transportadores intestinales para su absorción (Close et al., 2016; Wilson, 2015; Jeukendrup, 2013). 
Por lo que la fructosa, dependiente del transportador GLUT5, ha sido estudiada en combinación con otros HCO principalmente del grupo de oxidación rápida (dependientes de SGLT1), a esta combinación se le denomina MTC (Multiple Transportable Carbohydrates).

Entre los beneficios encontrados con el uso de MTC se encuentran:

Mayor absorción y mayores tasas de oxidación: El transporte no competitivo de glucosa y fructosa da como resultado una mayor entrega total de $\mathrm{HCO}$ a la circulación y una mayor oxidación en el músculo $\left(\mathrm{O}^{\prime}\right.$ Brien \& Rowlands, 2011; Jeukendrup, 2013). La oxidación de los HCO aumenta del 20 al $50 \%$ con el uso de MTC comparado con el consumo único de glucosa, además se ha demostrado una mayor eficiencia de oxidación con MTC, lo cual indica que una mayor proporción de los HCO consumidos son oxidados, siendo menor el residuo en el intestino (Jeukendrup, 2013; Stellingwerff \& Cox, 2014).

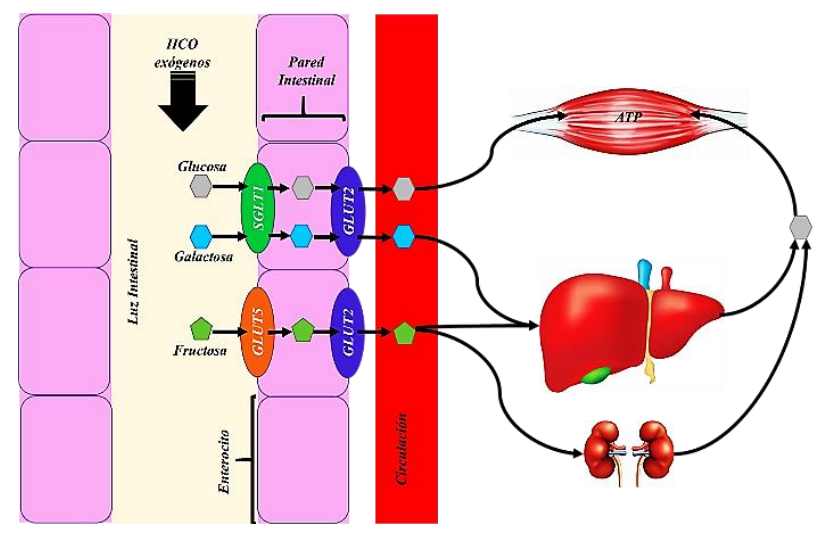

Figura 1 Absorción y oxidación de la glucosa, galactosa $\mathrm{y}$ fructosa

Fuente: Jeukendrup, 2013; Tappy y Rosset, 2017

Nota: La glucosa y galactosa dependen del transportador SGLT1 para su absorción en el enterocito, mientras que la fructosa requiere de GLUT5; el GLUT2 les proporciona una vía de salida común del enterocito a la circulación sanguínea. Una vez absorbida, la glucosa puede ser oxidada directamente por el musculo, por otro lado, la galactosa y la fructosa deben ser convertidos en glucosa principalmente por el hígado

Aumento del vaciado gástrico y absorción de líquidos: En varios estudios se ha demostrado que la administración de líquidos durante el ejercicio mejora con MTC en comparación con $\mathrm{HCO}$ de un solo transportador.
En teoría, el aumento en la absorción de HCO podría aumentar la absorción de agua al crear un gradiente osmótico favorable a través de la barrera celular epitelial, así como al aumentar el cotransporte de agua mediante el acoplamiento SGLT1 (Wilson, 2015; Jeukendrup, 2013).

Disminución del malestar gastrointestinal: Como resultado de un vaciado gástrico más rápido y una mayor absorción, la mayoría de los estudios también han reportado menos problemas gastrointestinales con MTC en comparación con una una sola fuente de HCO. Se cree que tener una alta eficiencia de absorción y oxidación de $\mathrm{HCO}$ ayuda a reducir la acumulación de $\mathrm{HCO}$ en el tracto gastrointestinal $\mathrm{y}$, de esta forma, se reduce también el malestar gastrointestinal durante el ejercicio (Roberts, Tarpey, Kass, Tarpey, \& Roberts, 2014; Wilson \& Ingraham, 2014; Sareban et al., 2016; Jeukendrup, 2013; Stellingwerff \& Cox, 2014; de Oliveira \& Burini, 2014).

Disminución de la fatiga y mejora del rendimiento: La mejora del rendimiento con el uso de MTC se atribuye principalmente al aumento en la absorción y oxidación de HCO, lo cual genera un mayor aporte de energía y retrasa la fatiga, sin embargo, también juega un papel importante la reducción de las molestias gastrointestinales (Stellingwerff \& Cox, 2014; de Oliveira \& Burini, 2014). En algunos estudios la ingestión de MTC ha resultado en calificaciones más bajas de esfuerzo percibido en comparación con la ingestión de $\mathrm{HCO}$ individuales (O’Brien \& Rowlands, 2011). De la misma forma en protocolos contrareloj o "time trials" (prueba para evaluar el rendimiento que consiste en recorrer una distancia determinada en el menor tiempo posible), se ha demostrado en repetidas ocasiones que el consumo de MTC disminuye el tiempo de finalización de la prueba respecto al consumo individual de HCO (Baur et al., 2014; Roberts et al., 2014; Wilson \& Ingraham, 2014). Es importante mencionar que para obtener beneficios adicionales con el consumo de MTC respecto a $\mathrm{HCO}$ individuales se necesitan tasas de ingesta superiores a 60-70 g / hora de HCO que permitan la saturación del transportador SGLT1, ya que cuando se consumen MTC a velocidades inferiores a $60 \mathrm{~g} /$ hora la oxidación de HCO es igual al consumo de $\mathrm{HCO}$ individuales. 
Cabe mencionar también que el efecto de los MTC es consistente en hombres y mujeres, en diferentes temperaturas ambientales, y entre ciclismo y carrera (Stellingwerff \& Cox, 2014).

\section{Agradecimiento}

Ricardo López Solís fue financiado por una beca del Programa Nacional de Posgrados de Calidad (PNPC) del Consejo Nacional de Ciencia y Tecnología (CONACYT), México.

\section{Conclusiones}

Debido a la alta demanda de suplementos nutricionales para la mejora del rendimiento, generada por el auge de los deportes de resistencia y el aumento en el número de atletas, existen en el mercado una gran variedad de suplementos ricos en $\mathrm{HCO}$, por sus efectos comprobados. Esto hace importante el entendimiento de los mecanismos de acción de los HCO en el organismo, para brindar un asesoramiento nutricional individualizado para los atletas de resistencia, tomando en cuenta la duración del ejercicio y los diferentes tipos de $\mathrm{HCO}$, y buscando un equilibrio entre la ingesta de HCO y el confort intestinal.

\section{Referencias}

Baur, D. A., Schroer, A. B., Luden, N. D., Womack, C. J., Smyth, S. A., \& Saunders, M. J. (2014). Glucose-fructose enhances performance versus isocaloric, but not moderate, glucose. Medicine \& Science in Sports \& Exercise, 46(9), 1778-1786.

Bompa, T., \& Buzzichelli, C. (2015). Periodization Training for Sports $\left(3^{\mathrm{a}}\right.$ ed.). Champaign, IL, Estados Unidos de América: Human Kinetics.

Campbell, B., Wilborn, C., La Bounty, P., Taylor, L., Nelson, M. T., Greenwood, M., ... Kreider, R. B. (2013). International Society of Sports Nutrition position stand: energy drinks. Journal of the International Society of Sports Nutrition, 10(1), 1.

Close, G. L., Hamilton, D. L., Philp, A., Burke, L. M., \& Morton, J. P. (2016). New strategies in sport nutrition to increase exercise performance. Free Radical Biology and Medicine, 98, 144-158. de Oliveira, E., \& Burini, R. (2014). Carbohydrate-dependent, exercise-induced gastrointestinal distress. Nutrients, 6(10), 41914199.

Díaz, F. J., Rodríguez, L., \& Rodríguez, J. J. (2017). Axiología y actividad física: el maratón como escenario de valores, significados y emociones. Revista Edu-física, 9(19), 94-111.

Fuchs, C. J., Gonzalez, J. T., \& van Loon, L. J. (2019). Fructose co-ingestion to increase carbohydrate availability in athletes. The Journal of physiology, 597(14), 3549-3560.

Instituto Nacional de Estadística y Geografía (México). (2013). Módulo de práctica deportiva y ejercicio físico 2013: MOPRADEF: Diseño conceptual/ Insituto Nacional de Estadística y Geografía. México.

Jeukendrup, A. E. (2011). Nutrition for endurance sports: Marathon, triathlon, and road cycling. Journal of Sports Sciences, 29(1), 9199.

Jeukendrup, A. E. (2013). Multiple transportable carbohydrates and their benefits. Sports Science Exchange, 26(108), 15.

Jeukendrup, A. E. (2017). Training the gut for athletes. Sports Medicine, 47(1), 101-110.

Kellett, G. L., Brot-Laroche, E., Mace, O. J., \& Leturque, A. (2008). Sugar absorption in the intestine: the role of GLUT2. Annual Review of Nutrition, 28, 35-54.

O'Brien, W. J., \& Rowlands, D. S. (2011). Fructose-maltodextrin ratio in a carbohydrateelectrolyte solution differentially affects exogenous carbohydrate oxidation rate, gut comfort, and performance. American Journal of Physiology-Gastrointestinal and Liver Physiology, 300(1), G181-G189.

Ormsbee, M., Bach, C., \& Baur, D. (2014). Preexercise nutrition: The role of macronutrients, modified starches and supplements on metabolism and endurance performance. Nutrients, 6(5), 1782-1808.

Peinado, A. B., Rojo-Tirado, M. A., \& Benito, P. J. (2013). El azúcar y el ejercicio físico: su importancia en los deportistas. Nutrición hospitalaria, 28, 48-56.

LÓPEZ-SOLÍS, Ricardo, CAMACHO-DÍAZ, Brenda Hildeliza, CAMPOS-MENDIOLA, Roberto y ORTÍZ-RODRÍGUEZ, María Araceli. Efecto del consumo de hidratos de carbono en los deportes de resistencia. Revista de Didáctica Práctica. 2019 
Peniche, C. (2011). Hidratos de carbono. En J. de León \& N.L. García (Eds.), Nutrición aplicada al deporte (pp. 13-37). Ciudad de México, México: McGraw-Hill Educación.

Pöchmüller, M., Schwingshackl, L., Colombani, P. C., \& Hoffmann, G. (2016). A systematic review and meta-analysis of carbohydrate benefits associated with randomized controlled competition-based performance trials. Journal of the international society of sports nutrition, 13(1), 27.

Roberts, J. D., Tarpey, M. D., Kass, L. S., Tarpey, R. J., \& Roberts, M. G. (2014). Assessing a commercially available sports drink on exogenous carbohydrate oxidation, fluid delivery and sustained exercise performance. Journal of the International Society of Sports Nutrition, 11(1), 8.

Run México. (2019). Reporte anual | 2018 Las carreras en México: Datos, números y estadísticas. México.

Sareban, M., Zügel, D., Koehler, K., Hartveg, P., Zügel, M., Schumann, U., ... \& Treff, G. (2016). Carbohydrate intake in form of gel is associated with increased gastrointestinal distress but not with performance differences compared with liquid carbohydrate ingestion during simulated long-distance triathlon. International journal of sport nutrition and exercise metabolism, 26(2), 114122. 